\title{
TOPOLOGY OPTIMIZATION BASED DESIGN OF LIGHTWEIGHT AND LOW VIBRATION GEAR BODIES
}

\author{
Ramadani, R. ${ }^{*}$; Belsak, A.** Kegl, M.**; Predan, J. ${ }^{* *} \&$ Pehan, S. \\ *University of Prishtina, Faculty of Mechanical Engineering, Sunny hill, p.n., 10000 Prishtina, Kosovo \\ ${ }^{* *}$ University of Maribor, Faculty of Mechanical Engineering, Smetanova 17, 2000 Maribor, Slovenia \\ E-Mail: riad.ramadani@uni-pr.edu, ales.belsak@um.si,marko.kegl@um.si,jozef.predan@um.si, \\ stanislav.pehan@um.si
}

\begin{abstract}
This article presents a new approach aiming to reduce gear vibration and weight by modifying its body structure. The primary objective was to reduce vibration and noise emission of spur gears. For this purpose, a solid gear body was replaced by a lattice structure, which was expected to raise the torsional compliance of the body. The lattice structure was configured and optimized by a FE-based topology optimization software. For experimental purposes, the optimized gear was produced from Titanium alloy Ti-6Al-4V ELI using Selective Laser Melting technique. In the tests, the sound pressure of various running gear pairs was measured in order to estimate and compare the properties of a solid gear, of a lattice gear, and of a lattice gear, filled with polymer to increase the structural damping. It was experimentally confirmed that the cellular lattice structure of a gear body and addition of a polymer matrix may significantly reduce the vibration.

(Received in July 2017, accepted in November 2017. This paper was with the authors 1 month for 1 revision.)
\end{abstract}

Key Words: Gear Body, Lightweight Lattice Structure, Topology Optimization, Stress Reduction, Vibration Reduction

\section{INTRODUCTION}

Gears are crucial parts of most mechanical power transmissions. With the increasing demands on weight, speed, and running conditions, both, lightweight design and reduction of vibrations and noise gain an ever increasing importance. Vibrations are typically induced by external influences, for example, from changing torque and speed. Apart from this, there are always internally generated vibrations, for example, those caused by tooth meshing [1]. Due to the repeated stiffness changing during the tooth meshing, each toothed gear ring becomes an internal generator of vibrations.

Most of the recent research work, related to gear noise and vibration reduction, is focused on tooth geometry. For example, to smooth the meshing the authors of [2, 3] focused on tooth profile modification. Furthermore, sophisticated optimization procedures to improve the tooth flank were employed by the authors of [4-6] in order to gain a calmer gear run. Unfortunately, it seems that irrespective of any modifications of the tooth's profile or its flank, tooth meshing will always remain a vibration generator [7-9]. Besides of this, a tooth profile modification may also lead to an increase of tooth flank stresses [10,11].

To a somewhat lesser extent, related research work focuses on gear body, which is considered as a vibration transmitter that offers a potential for improvement. The authors of $[12,13]$ suggested to fill relatively large holes, which are located around the gear body, with a powder material. In that way, the gear body becomes a barrier that hinders the vibration propagation to some extent.

Quite some gear body modification studies are targeting exclusively the weight of the body. Of course, making the gear body slimmer and weaker reduces weight and saves material. Unfortunately, in general, the dynamics of the gear drive might not change much or can become even worse $[14,15]$. Thinner walls and induced holes simply do not reduce significantly the vibration transmission from the toothed ring to other parts of the drive. 
The aim of this paper is to take advantage of the recent production technologies and the accompanying emerging software in order to design the gear body so that it will fulfil strength-related requirements but will also reduce and dissipate, both, internally and externally induced vibrations. The basic idea is to produce a body structure that is lighter as a solid body, sufficiently firm in term of load-carrying capacity, flexible in the tangential direction, and suitable to be filled with viscous plastic material.

In this work the gear body is designed as a cellular lattice structure. In this way, the vibrations travelling paths automatically become longer, which is expected to have a positive influence on vibrations dissipation due to internal material friction. Additionally, lightweight design is expected to be more flexible or compliant in the tangential direction. This is also expected to have a positive influence on transmitting the vibrations from the gear ring to the hub.

In general, a cellular lattice structure is assembled by repeating cell patterns, which, in turn, are lattice structures. Until recently, lattice structures could only be designed or optimized in a relatively modest way because of very limited production capabilities. This has changed a lot by emerging additive manufacturing technologies. So, available software is now catching up and currently it is already possible to design useful lattice structures. Unfortunately, the related FEA models can typically employ only beam finite elements [16]. This automatically means that stress concentrations, emerging at lattice member joints, cannot be removed effectively by structural optimization. Since stress concentrations removal is of paramount importance for load carrying parts, one really has to look for a procedure that enables effective numerical optimization of the whole structure, including the very details around lattice member joints, Fig. 1. To design and shape the joints properly, the underlying numerical model must be based on full 3D solid finite elements.

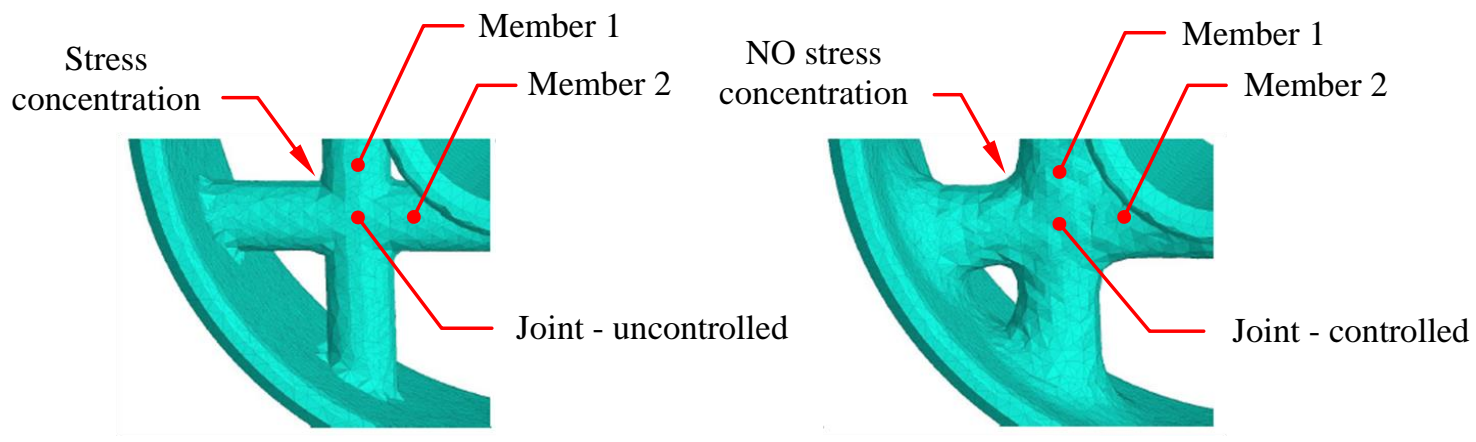

Figure 1: Details of the lattice structure.

In this work CAESS ProTOp [17] was engaged to configure and optimize the lattice structure of the gear body. This is a high-performance topology optimization software based exclusively on full 3D solid finite elements. In this way it was actually possible to get a good lattice design with precisely shaped lattice member joints and without any stress concentrations. The obtained optimal spur gear was then produced by Selective Laser Melting technique and tested on a closed loop test rig. Its dynamic response and properties were compared to a solid body gear. It turned out that the lattice body is capable to lower the sound pressure emitted by the gear, while preserving its strength properties.

\section{LATTICE STRUCTURE OF A GEAR BODY}

It is commonly recognized that lattice structures may offer various advantages in the context of load-carrying parts design. Only recently however, such design became technologically admissible due to new emerging production technologies and optimization software. 


\subsection{Possible benefits of a lattice structure design}

In line with the traditional design guides, machine parts are often made too heavy or exhibit properties that might be better. Machine parts such as gears, consist mostly of large areas in which the stress levels are wastefully low and of some exposed spots, in the vicinity of which stresses are close to the allowable limit. Moreover, a solid body is a perfect translator and transmitter of vibrations and it often represents a substantial contribution to the total weight of the machine part. A replacement of a solid body by a lattice structure can therefore be expected to bring benefits in the sense of weight reduction and vibration behaviour.

The gears, in general, are actually excellent examples when it comes to introducing lattice structures into the design process. Namely, traditionally they are mostly too heavy, they are a source of vibration, they transmit and translate vibrations, and their body is typically loaded at stress levels far below the admissible limits. A gear consists of a hub and toothed ring which are connected by a gear body. The primary roles of the gear body are to position the other two parts and to transmit the loading. Often, a solid gear body would be an over-dimensioned part of the gear. So, various weight-reducing designs are currently engaged if this is acceptable by technological and economical constraints, Fig. 2.
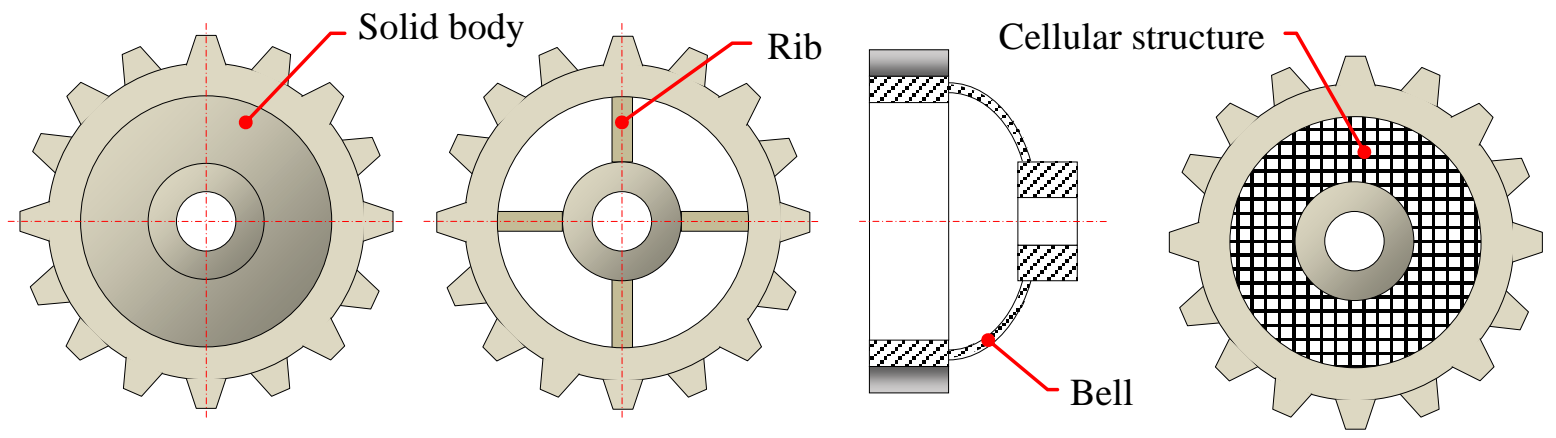

Figure 2: Some gear body weight reduction options.

The most common approach to gear body modification is to introduce ribs distributed circumferentially. The ribs themselves can be shaped into many various forms. Using a thinwalled gear body can also be a very effective approach for weight reduction, especially, if the body is shaped into a bell form. Considering the vibrations, however, thin walls can lead to harmful side effects due to rising vibration emission. In this view, the introduction of a lattice structure could be expected to bring more or less positive effects only: reduced weight, lower noise emission, and lower vibration transmission. Although it will probably be tangentially stiffer than a body with conventional ribs, it will still be more compliant than a thin-walled body.

On the negative side, an introduction of a lattice design raises concerns about possible problems related to load carrying capability of a gear. Eventual stress concentrations and consequent crack initiations could lead to structural failure. Therefore, to recover structural robustness and reliability, a lattice structure has to be optimized by all means. The objective should be to remove stress concentrations completely and to lover the stress levels to the lowest attainable level.

\subsection{Optimization of a lattice structure}

Topology optimization of lattice structures is a relatively new and emerging field that gained attention with the development of new additive manufacturing technologies. According to [18] topology optimization of a lattice structure modelled by solid finite elements differs from conventional topology optimization in one crucial step: configuring of the optimization 
domain. This configuration step enforces the chosen lattice structure on a usual solid domain meshed by solid finite elements and prevents the optimizer to add material outside of the domain of the lattice structure. At this point it might be worth noting that lattice structure enforcement automatically results in a sub-optimal design from the mathematical point of view. It is expected however, that reliability and robustness of an optimized lattice structure will be better than a corresponding optimized solid model. Theoretically, this could be even further improved by optimizing also lattice cell parameters. Currently, however, it seems that this option is not yet supported by existing software.

In this work the domain configuring and optimization was done by CAESS ProTOp which redistributes the material based on a material function defined by nodal material parameters $p_{i}$ in the range $[0,1]$. These are used to define computational values of material density and modulus of elasticity as follows:

$$
\rho_{i}=p_{i} \rho_{0}, \quad E_{i}=p_{i} E_{0}
$$

where $\rho_{0}$ and $E_{0}$ are the actual density and elastic modulus of the engaged material, while $\rho_{i}$ and $E_{i}$ are the corresponding computational values used for the numerical analysis. In this way a certain region of the design space tends to become void as $p_{i} \rightarrow 0$ or full material as $p_{i} \rightarrow 1$.

The definition of the objective function depends on the desired structural properties. If removed stress concentrations and low stress levels within a statically loaded structure are desired, the objective function $f$ to be minimized is typically defined as the total strain energy of the structure:

$$
f=1 / 2 \int_{B} \sigma: \varepsilon d V
$$

where $\sigma$ denotes the Cauchy stress tensor, $\varepsilon$ is the small-strain tensor, and integration has to be done over the whole structural domain $B$. The term $\sigma: \varepsilon$ denotes the double inner product of both tensors; in index notation with summation convention, this product is written as $\sigma_{i j} \varepsilon_{i j}$.

During the optimization process, the material is redistributed within the design domain so that the objective function $f$ is minimized. In case of a lattice structure, this material redistribution can only happen within the lattice domain, which is a subset of the whole (solid) design domain. A typical constraint in this process is some prescribed volume fraction; this constraint limits the total volume of the final optimized structure.

\section{GEAR BODY DESIGN PROCESS}

In this work a spur gear, as specified in Table I, was addressed. The numerical FEA-ready model of the gear with a full solid gear body was prepared within SIMULIA Abaqus [19]. The diameter of the fixing hole was $25 \mathrm{~mm}$ and the nominal torque adopted was $200 \mathrm{Nm}$. The gear was meshed with linear hexahedral elements of type C3D8, which are the eight-node linear brick elements. Hexahedral elements were used in the three-dimensional analysis because the generated mesh exhibited symmetries which influence very positively the symmetries of the final design. Because of the planned topology optimization the mesh has to be relatively fine and consequently the number of elements was relatively high; the mesh contained a total of 2918412 elements. To influence positively the design symmetries and cyclic patterns, a total of 68 static load cases were defined: one load case per one side of each tooth. The applied load was computed from the nominal torque, which was converted into pressure of $p=618 \mathrm{MPa}$ and applied to a tooth flank. The loading point was the outer single meshing point. The inner surface of the hub was fixed by adequate displacement constraints. This FEA model was then imported into CAESS ProTOp for further design configuring and optimization. 
Table I: Basic data of a spur gear.

\begin{tabular}{|l|c|}
\hline Data & Value \\
\hline Number of teeth & 34 \\
\hline Normal module & $2.5 \mathrm{~mm}$ \\
\hline Pressure angle & $20^{\circ}$ \\
\hline Width & $10 \mathrm{~mm}$ \\
\hline Pitch diameter & $85 \mathrm{~mm}$ \\
\hline Base diameter & $79.874 \mathrm{~mm}$ \\
\hline Root diameter & $78.558 \mathrm{~mm}$ \\
\hline Tip diameter & $90 \mathrm{~mm}$ \\
\hline Young`s modulus & $206000 \mathrm{MPa}$ \\
\hline Poisson`s ratio & 0.3 \\
\hline
\end{tabular}

\subsection{Configuring of the gear body}

The first step in preparing an adequate optimization model is to define the properties of various subdomains of the part. In our case the hub ring and the toothed ring must remain unchanged during optimization; so they are defined as fixed domains. On the other hand, the gear body will be defined as the design domain, free for optimization. The considered spur gear with chosen fixed and free domains is presented in Fig. 3.

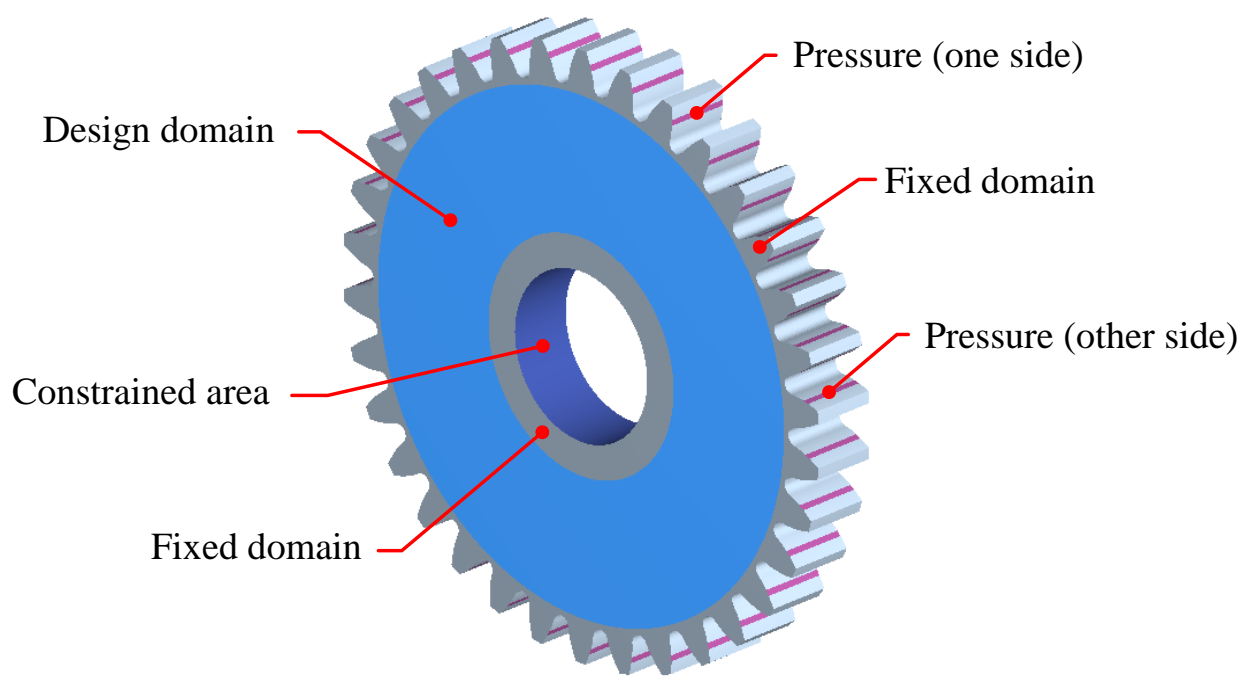

Figure 3: Free and fixed domain of the considered spur gear.

In the second step of preparing the optimization model, the gear body had to be configured in order to replace the full solid design with a lattice design. For this purpose two different lattice cell types have been used: a cube diagonal lattice cell and a plane diagonal lattice cell, Fig. 4.

The inner part of the gear body was configured by using cube diagonal cells defined in a cylindrical coordinate system, which is defined by radius and angle; the radial dimension of the cube diagonal cell was chosen to be $6 \mathrm{~mm}$ and the angle was 10.590 degrees. The width of the cube diagonal lattice cell was $7 \mathrm{~mm}$. The diameter of the cell diagonals was allowed to change within the range from 0.8 to $2 \mathrm{~mm}$. 

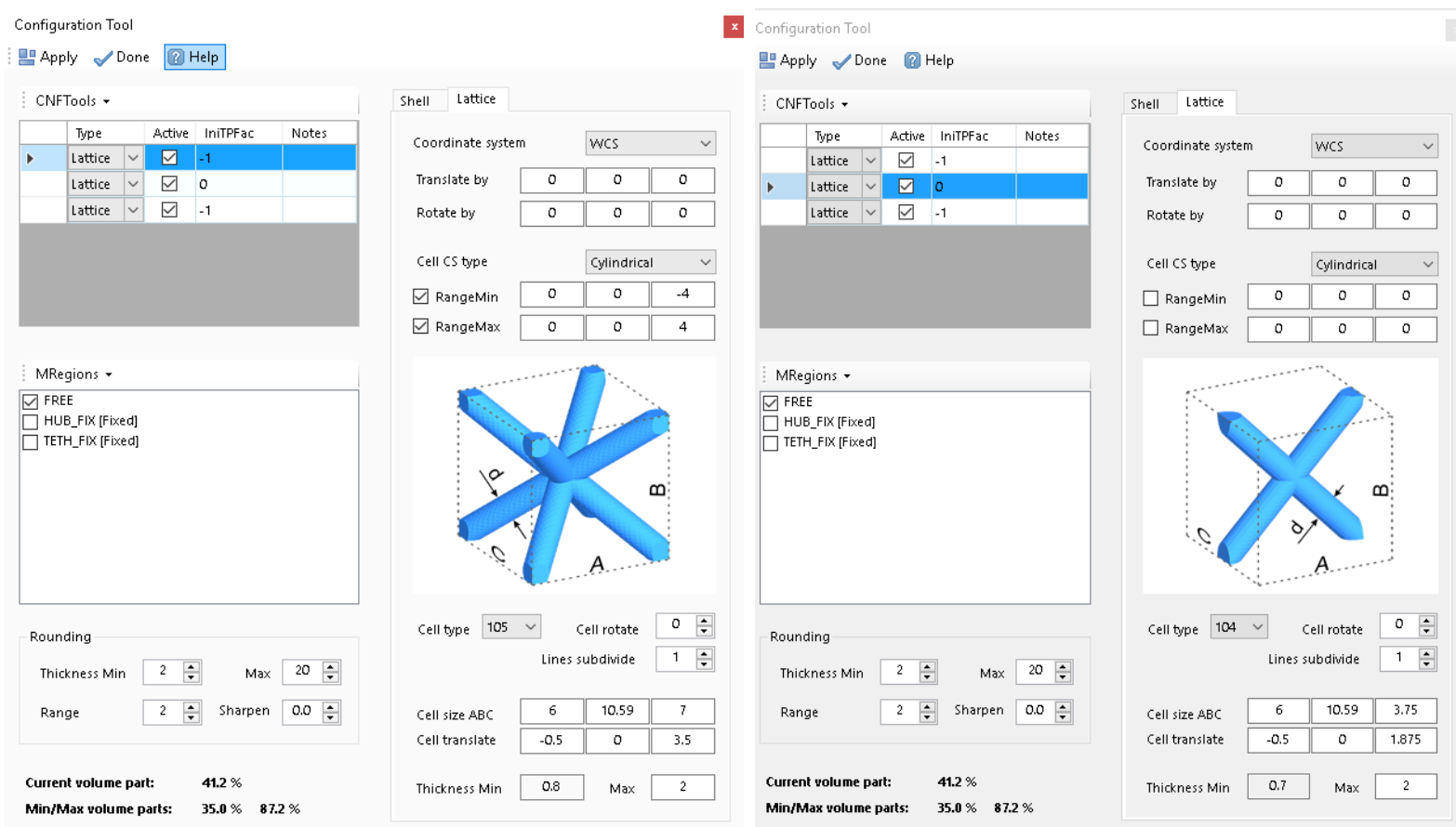

Figure 4: Configuration tool for the cube diagonal lattice and diagonal lattice structure.

The outer part of the gear body was configured by the plane diagonal lattice cell. The radial dimension of this cell was chosen to be $6 \mathrm{~mm}$ and the angle was 10.59 degrees. The width of the cell was $3.75 \mathrm{~mm}$ and the diameter its diagonals can vary from 0.7 to $2 \mathrm{~mm}$.

The outermost ring of the free domain was configured as a solid domain in order to provide sufficient stiffness for the gear ring. This step finalized the configuring of the gear body. At this stage the volume part of the body was $41.2 \%$ of the full solid design. This initial configured design is presented in Fig. 5.

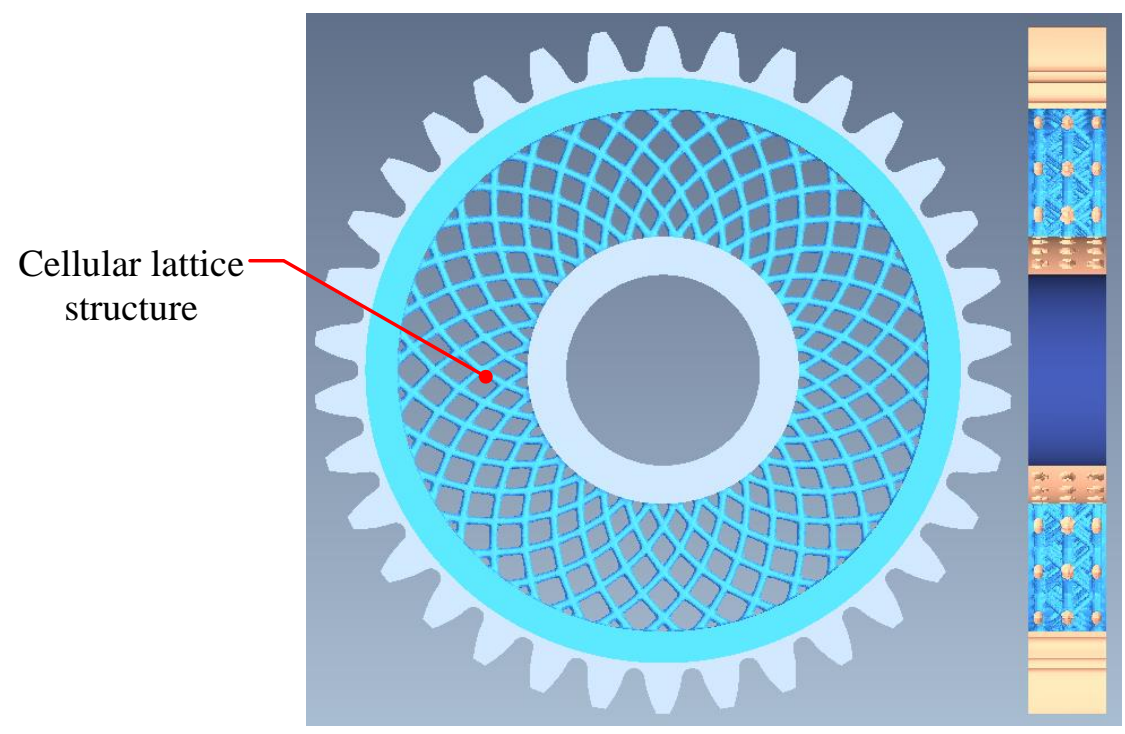

Figure 5: Initial design of the spur gear body, configured as a lattice structure.

\subsection{Optimization of the gear body structure}

The optimization procedure was an iterative process done by CAESS ProTOp [17]. Each iteration requires one full finite element analysis per load case and one computation of topology parameters; i.e. each iteration requires 68 full FE analyses. ProTOp performs 
structural topology optimization by sequential design steps which minimize the total strain energy of the structure [17].

The stresses corresponding to the initial configured design with a volume part of $41.2 \%$ exhibited peak levels about $590 \mathrm{MPa}$ (Fig. 6; gear tooth region), while the stresses within the lattice structure reached maximum levels about $400 \mathrm{MPa}$ (Fig. 6; detail view; yellow colour). The structure was then optimized and the volume part of the free domain was increased from $41.2 \%$ up to $49.3 \%$. Surprisingly this also reduced stresses within the tooth region, which now peaked to about $550 \mathrm{MPa}$. More importantly, however, are the stress levels within the optimized lattice structure. These stresses dropped dramatically with maximum stress levels reaching now only about $150 \mathrm{MPa}$.

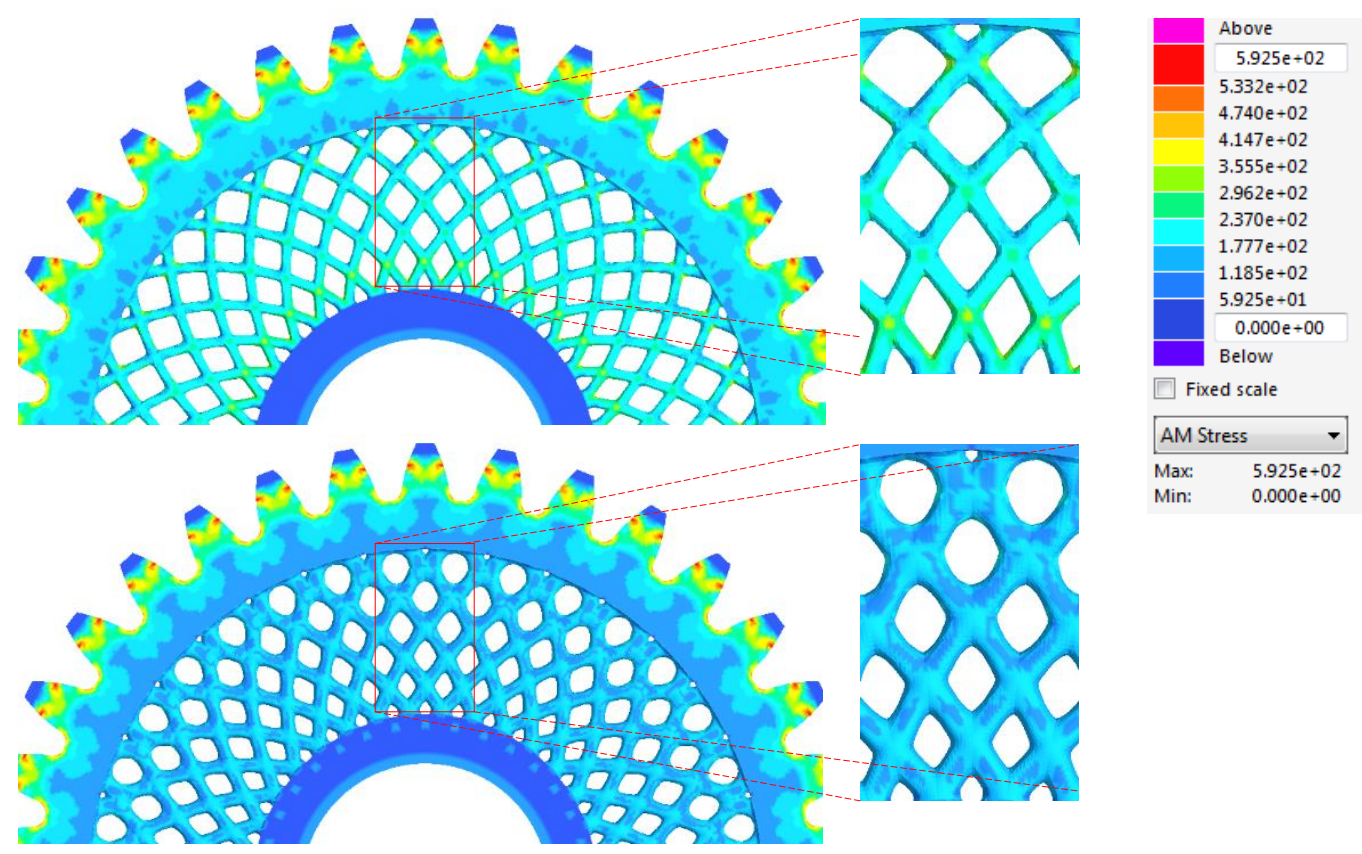

Figure 6: Von Misses stress field of the initial and optimized lattice structure.

In order to get a better view of the stress field within the lattice body structure, a cross section of the initial and optimized lattice is presented in Fig. 7.
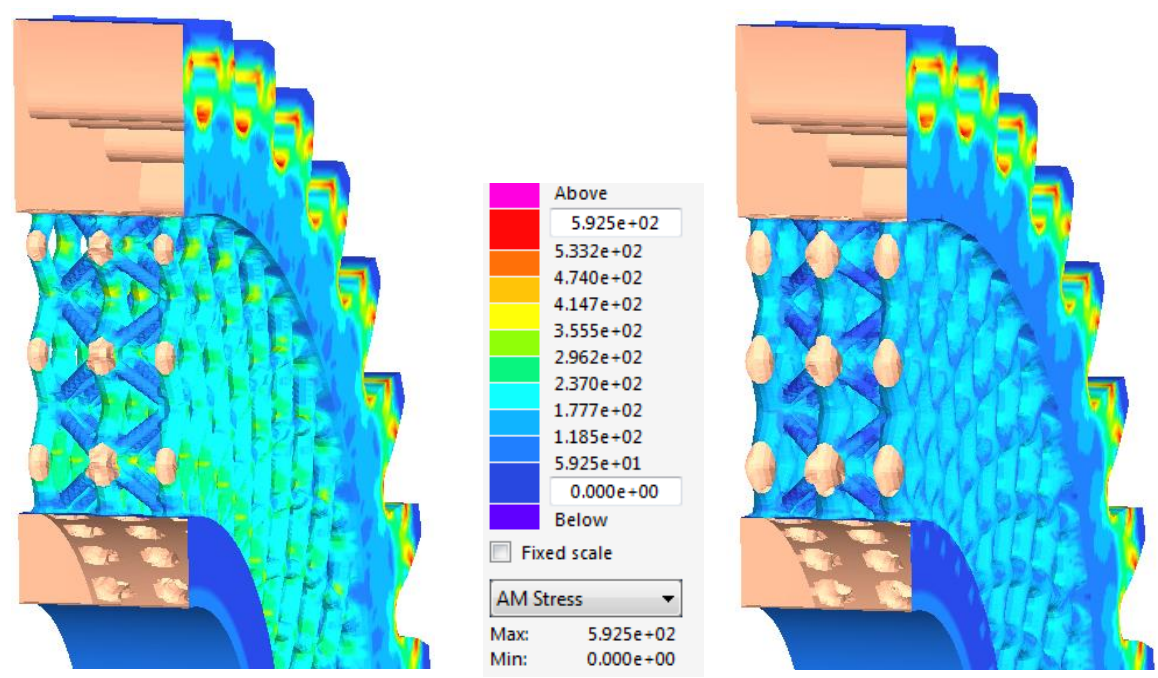

Figure 7: A cross section of initial and optimized lattice structure; von Misses stress field. 
The optimized gear was planned to be tested experimentally. The production of such a part is currently possible only by engaging additive manufacturing technologies. A titanium alloy Ti-6Al-4V ELI and Selective Laser Melting technique were chosen to produce the spur gear with the optimized lattice. SLM allows a part to be built additively, layer by layer, from an adequate powder metal [20,21]. The parameters that have been used to print the gear were: laser power $75 \mathrm{~W}$; scan speed $600 \mathrm{~mm} / \mathrm{s}$; layer thickness 35 microns. The teeth flank and the inner surface of the hub were grinded in the same tolerances as the gear with a solid body.

The titanium printed gear is shown in Fig. 8.

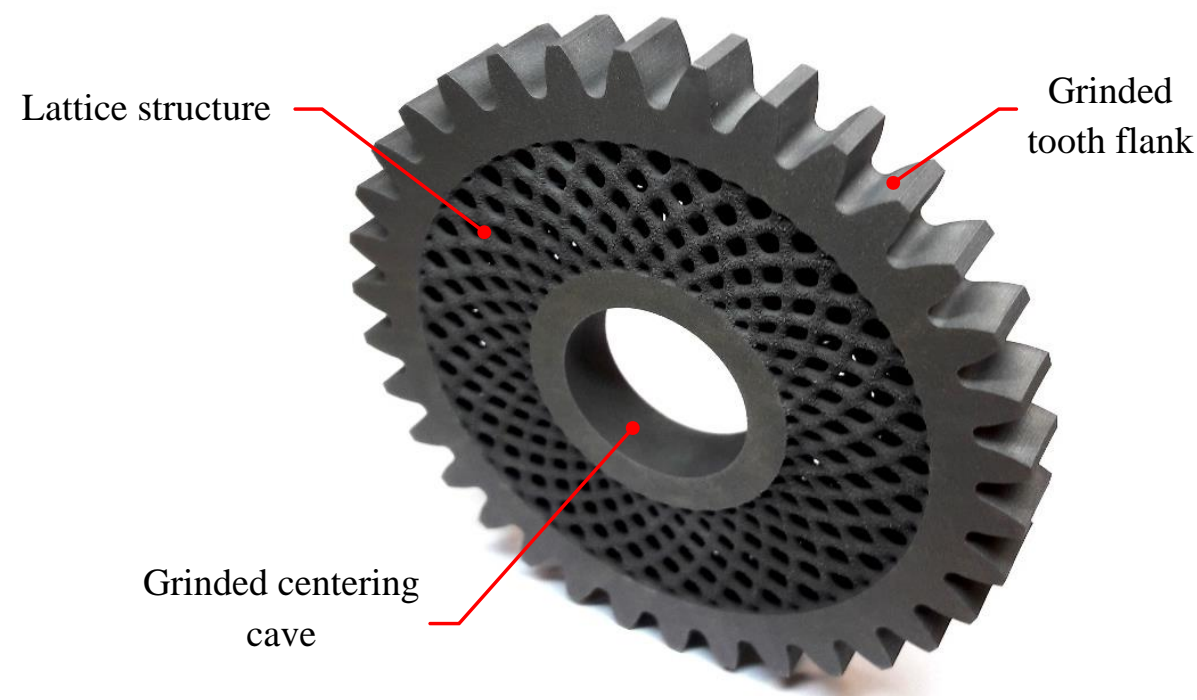

Figure 8: Photo of the titanium printed gear.

\section{DYNAMIC BEHAVIOUR OF THE OPTIMIZED LATTICE GEAR}

To evaluate vibration behaviour of the optimized lattice structure gear, the sound pressure of two engaged gear pairs, operating under some specified load, has been measured. The first gear pair, which acts as a reference pair, consists of two solid body gears. On the other hand, the second gear pair consists of one solid body gear which is engaged with the optimized lattice structure gear.

To measure the dynamic behaviour of the gear pair under realistic loading conditions, a new test rig with closed loop has been conceived, designed, and produced [22]. This was necessary in order to assure a test rig with the highest possible rigidity and adequate lubrication condition. For convenience the housing of the rig was designed to be partly transparent and easy removable. The test rig is equipped with two gear pairs: with the tested one and with the driving one. The axial distance of the gear pairs is $90 \mathrm{~mm}$. The gears have been run and tested at different speeds, but all of the presented diagrams have been recorded at $n=310 \mathrm{RPM}$ at applied torque of $T=60 \mathrm{Nm}$. The sound pressure was recorded by the AP 7046 microphone that was located close to the gears. The signal was processed by the National Instruments NI PXI 4472 system. The final processing of the signal was done by specially developed software based on LabView. By employing the fast Fourier transformation [23] the time signal of sound pressure has been converted to the frequency spectrum that was processed by adequate software based on LabView. The frequencies were analysed up to the $7 \mathrm{kHz}$, while the measured time signal was $0.2 \mathrm{~s}$ long.

The time signal of the reference gear pair is presented in Fig. 9, whereas its frequency spectrum is given in Fig. 10. The time signal for the gear with lattice structure is presented in Fig. 11, whereas its frequency spectrum is shown in Fig. 12. The time signal for the gear with 
lattice structure filled with polymer inserted is presented in Fig. 13; its frequency spectrum is shown in Fig. 14.
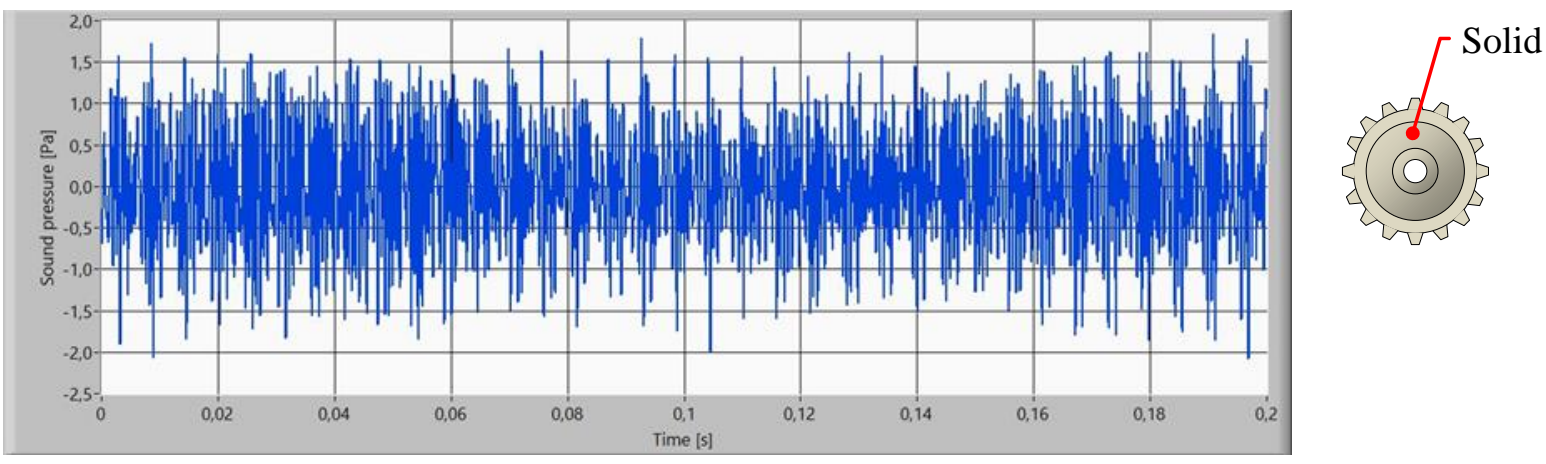

Figure 9: Time signal of sound pressure for the gear with a solid body.
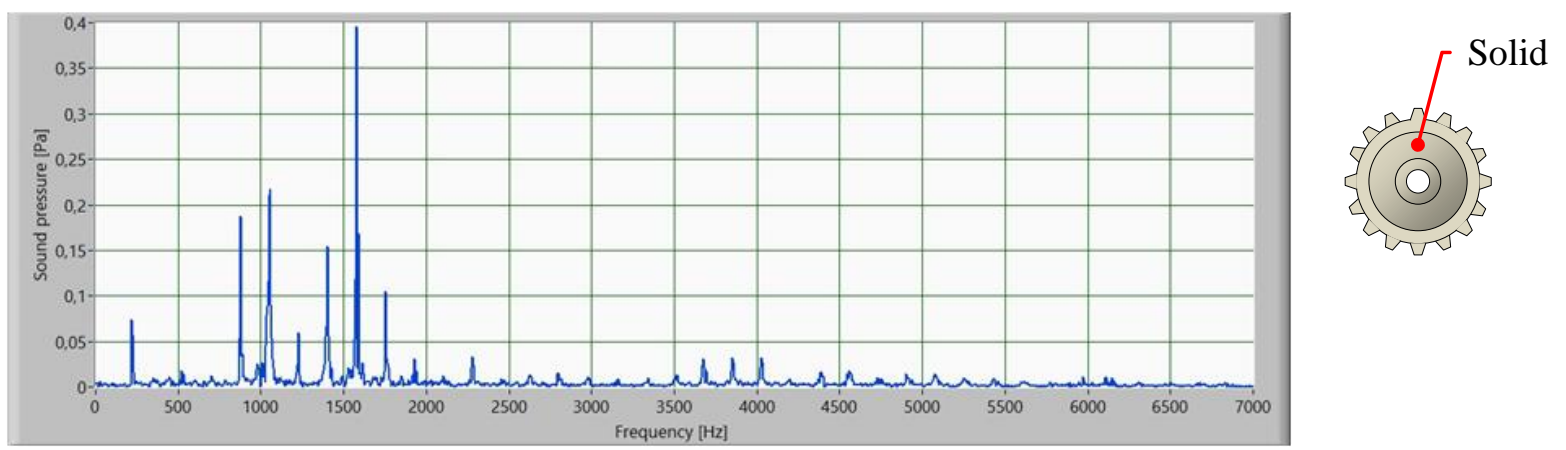

Figure 10: Frequency spectrum of sound pressure for the gear with a solid body.
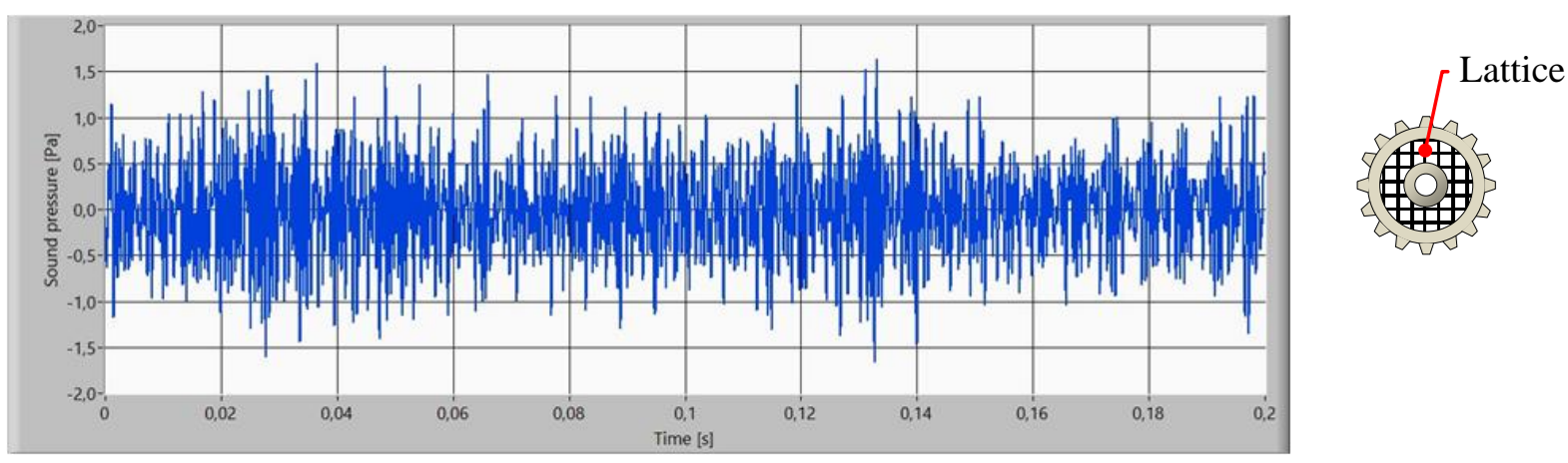

Figure 11: Time signal of sound pressure for the gear with the lattice structure.
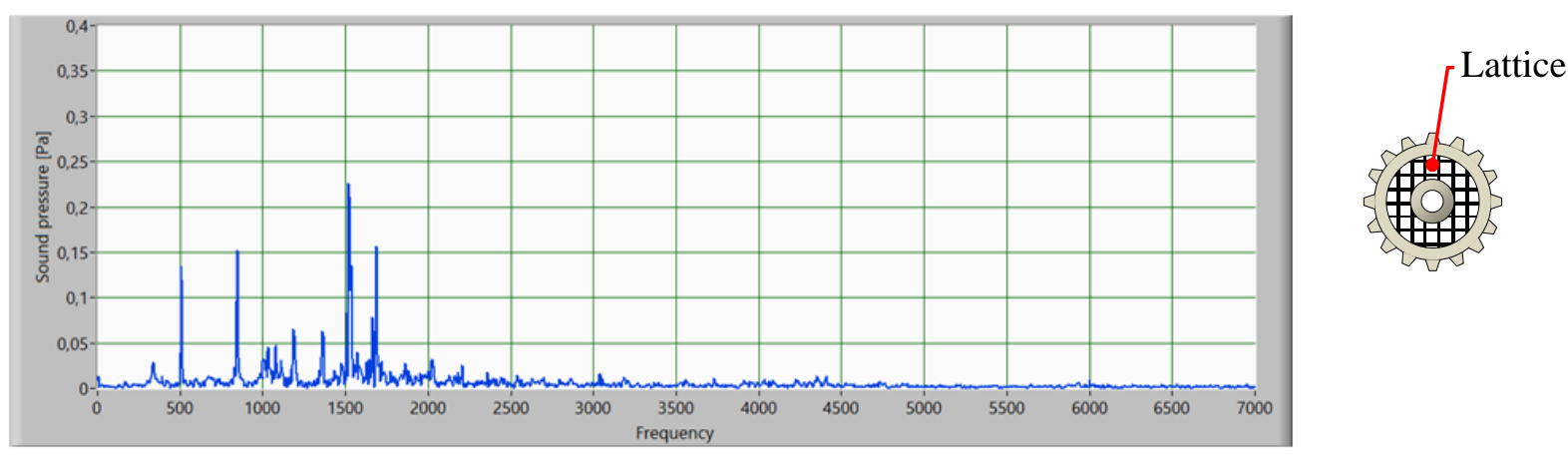

Figure 12: Frequency spectrum of sound pressure for the gear with the lattice structure. 

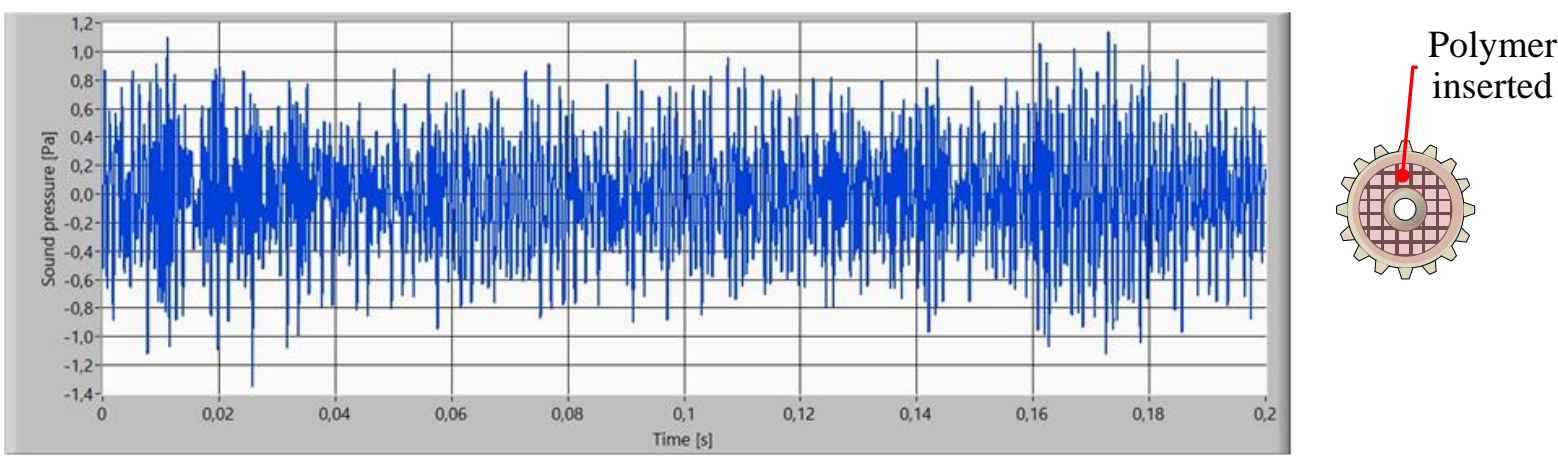

Figure 13: Time signal of sound pressure for the gear with lattice structure with polymer inserted.
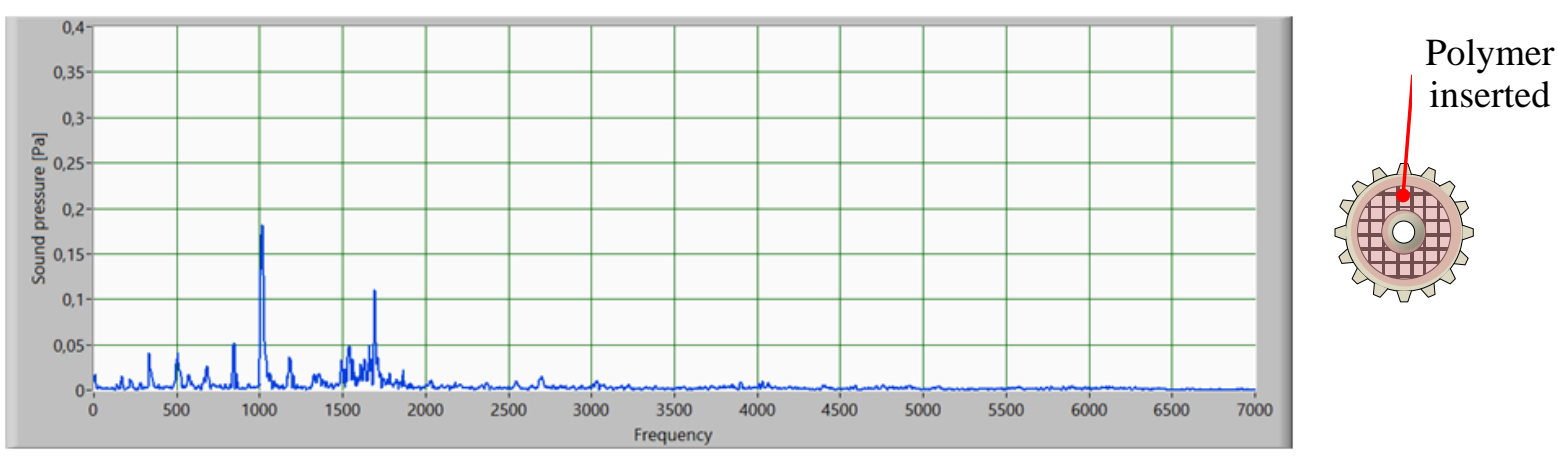

Figure 14: Frequency spectrum of sound pressure for the gear with lattice structure with polymer inserted.

From the frequency spectrum of the gear with full solid body, presented in Fig. 10, it is evident that the dominating amplitudes are achieved at the $8^{\text {th }}, 5^{\text {th }}, 4^{\text {th }}$ and $7^{\text {th }}$ harmonics of the gear meshing frequency; the highest pressure value was observed at the $8^{\text {th }}$ harmonics. The pressure values of the $9^{\text {th }}$ and higher harmonics are observable but relatively low.

In the frequency spectrum of the gear with lattice structure, presented in Fig. 12, the $8^{\text {th }}$ harmonics of fundamental gear meshing frequency is also the dominating one. The other distinctive pressure values appeared at the $9^{\text {th }}, 4^{\text {th }}$ and $2^{\text {nd }}$ harmonic. The pressure values of frequencies of $2 \mathrm{kHz}$ and more are practically negligible.

In the frequency spectrum of the gear with lattice structure with polymer inserted, presented in Fig. 14, the $5^{\text {th }}$ harmonics turned out to be the dominating one. The second largest pressure peak (with left sideband frequency) was observed at the $9^{\text {th }}$ harmonics. Again, the pressure values of frequencies of $2 \mathrm{kHz}$ and more are practically negligible.

The pressure peaks of the solid gear, lattice structure gear, and polymer filled lattice structure gear, are presented in Fig. 15.

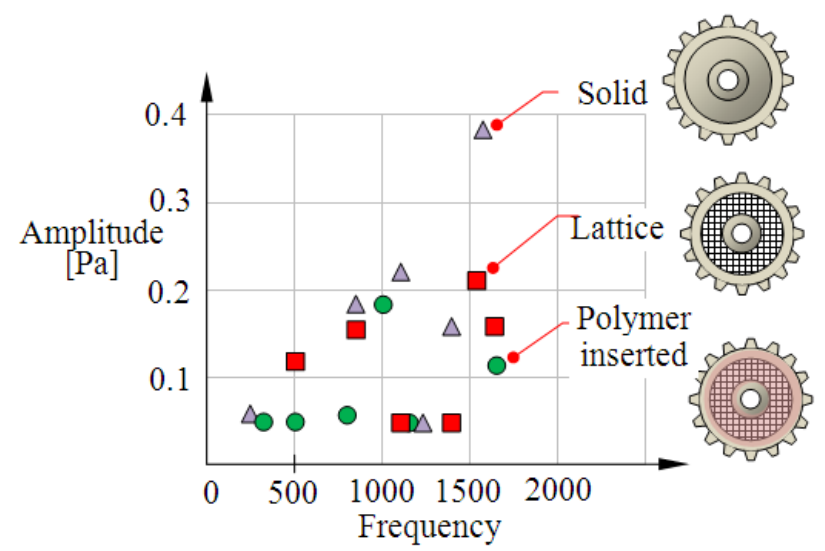

Figure 15: Pressure peaks of: solid, lattice structure, and polymer filled lattice structure gears. 
As shown in Fig. 15 the largest pressure amplitude was observed for the gear with a solid body; a pressure peak of around $0.39 \mathrm{~Pa}$ appeared at the frequency of $1575 \mathrm{~Hz}$. For the gear with lattice structure, the maximal pressure amplitude is substantially lower; a pressure peak of around $0.225 \mathrm{~Pa}$ appeared at the frequency of $1575 \mathrm{~Hz}$. A further reduction of the maximal pressure value was achieved by filling the lattice structure with a polymer. This gear exhibits a maximum of $0.18 \mathrm{~Pa}$ at the frequency of $1050 \mathrm{~Hz}$.

When the frequency spectrum is compared (Figs. 10, 12 and 14), it is evident that the peaks are higher and most clearly separated for the solid body gear. On the other side, the peaks are lower with more sidebands for the lattice structure body gears. This is especially true for the polymer filled gear. It is obvious that replacing the solid body by a lattice structure, some dominating frequency components practically either disappear or are shifted to lower frequency range. Obviously, lattice structure does quite a good job in reducing gear vibrations and the inserted polymer, which increases the structural damping, is also very helpful.

At this point it might be worth noting that besides of reduced vibrations, the optimized lattice body gear also exhibits a substantially reduced mass. Namely, the volume of the gear with a solid body is about $51130 \mathrm{~mm}^{3}$, while the volume of the gear with the optimized lattice structure is about $32570 \mathrm{~mm}^{3}$. Thus, the volume of the used material is reduced by around one third. The weight of the gear with a solid body is $0.40137 \mathrm{~kg}$, whereas the weight of the gear with the optimized lattice structure is $0.25567 \mathrm{~kg}$.

\section{CONCLUSION}

In this paper, the influence of gear body structure on gear vibration during operation has been investigated. The body was designed as a cellular lattice structure and topology optimization of this structure was employed in order to remove stress concentrations and to reduce the stress levels as much as possible. A spur gear with the optimized lattice structure was produced by using Selective Laser Melting technique. This gear was then used in experimental measurements of sound pressure during gear operation.

The results obtained clearly indicate that an adequate body structure may well result in significant reduction of gear vibrations. According to experimental results, a lattice structure gear body is an interesting option since it might notably reduce vibrations, besides of the obvious benefit of weight reduction. A lattice structure offers also a nice opportunity to be filled with an adequate polymer. According to the results, this may further reduce the vibrations.

The main disadvantage of a lattice structure is that it is a rather expensive choice since traditional manufacturing is not possible and so $3 \mathrm{D}$ printing technologies have to be engaged. Additionally, the teeth flank of a printed gear must be grinded which requires an extra production operation. Another, less obvious, point of concern is that currently printed materials are not very resistant against fatigue crack initiation and consequent structural failure. To mitigate this drawback, a printed part has to be optimized by all means in order to reduce the stresses as much as possible.

\section{ACKNOWLEDGEMENT}

The article was written under favourable circumstances of Faculty of Mechanical Engineering, University of Maribor, Slovenia. The authors want to thank Erasmus Mundus JoinEU-SEE PENTA scholarship program, Slovenian Research Agency (research core funding No. P2-0063) as well as CAESS company for the research support. 


\section{REFERENCES}

[1] Smith, J. D. (2003). Gear Noise and Vibration, $2^{\text {nd }}$ edition, Marcel Dekker, Inc., New York

[2] Kahraman, A.; Blankenship, G. W. (1999). Effect of involute tip relief on dynamic response of spur gear pairs, Journal of Mechanical Design, Vol. 121, No. 2, 313-315, doi:10.1115/1.2829460

[3] Liu, G.; Parker, R. G. (2008). Dynamic modeling and analysis of tooth profile modification for multimesh gear vibration, Journal of Mechanical Design, Vol. 130, No. 12, Paper 121402, 13 pages, doi:10.1115/1.2976803

[4] Bonori, G.; Barbieri, M.; Pellicano, F. (2008). Optimum profile modifications of spur gears by means of genetic algorithms, Journal of Sound and Vibration, Vol. 313, No. 3-5, 603-616, doi:10.1016/j.jsv.2007.12.013

[5] Faggioni, M.; Samani, F. S.; Bertacchi, G., Pellicano, F. (2011). Dynamic optimization of spur gears, Mechanism and Machine Theory, Vol. 46, No. 4, 544-557, doi:10.1016/j.mechmachtheory. 2010.11.005

[6] Ghosh, S. S.; Chakraborty, G. (2016). On optimal tooth profile modification for reduction of vibration and noise in spur gear pairs, Mechanism and Machine Theory, Vol. 105, 145-163, doi:10.1016/j.mechmachtheory.2016.06.008

[7] Abbes, M. S.; Fakhfakh, T.; Haddar, M. (2005). Gear box vibratory analysis using carrying, coupling and slave substructures, International Journal of Simulation Modelling, Vol. 4, No. 2, 67-75, doi:10.2507/IJSIMM04(2)2.043

[8] Chaari, F.; Hbaeib, R.; Fakhfakh, T.; Haddar, M. (2005). Dynamic response simulation of planetary gears by the iterative spectral method, International Journal of Simulation Modelling, Vol. 4, No. 1, 35-45, doi:10.2507/IJSIMM04(1)4.037

[9] Li, X. Y.; Zhang, Q. X.; Wang, N. N.; Zeng, Q. L.; Hidenori, K. (2017). Meshing simulation and strength calculation of a carburized gear pair, International Journal of Simulation Modelling, Vol. 16, No. 1, 121-132, doi:10.2507/IJSIMM16(1)10.376

[10] Marković, K.; Vrcan, Ž. (2016). Influence of tip relief profile modification on involute spur gear stress, Transactions of FAMENA, Vol. 40, No. 2, 59-70, doi:10.21278/TOF.40205

[11] Duhovnik, J.; Zorko, D.; Sedej, L. (2016). The effect of the teeth profile shape on polymer gear pair properties, Technical Gazette, Vol. 23, No. 1, 199-207, doi:10.17559/TV-20151028072528

[12] Xiao, W.; Li, J.; Wang, S.; Fang, X. (2016). Study on vibration suppression based on particle damping in centrifugal field of gear transmission, Journal of Sound and Vibration, Vol. 366, 6280, doi:10.1016/j.jsv.2015.12.014

[13] Xiao, W.; Huang, Y.; Jiang, H.; Jin, L. (2016). Effect of powder material on vibration reduction of gear system in centrifugal field, Powder Technology, Vol. 294, 146-158, doi:10.1016/ j.powtec.2016.01.038

[14] Li, S. (2008). Experimental investigation and FEM analysis of resonance frequency behavior of three-dimensional, thin-walled spur gears with a power-circulating test rig, Mechanism and Machine Theory, Vol. 43, No. 8, 934-963, doi:10.1016/j.mechmachtheory.2007.07.009

[15] Cai, Z.; Lin, C. (2017). Dynamic model and analysis of nonlinear vibration characteristic of a curve-face gear drive, Strojniski vestnik - Journal of Mechanical Engineering, Vol. 63, No. 3, 161-170, doi:10.5545/sv-jme.2016.3859

[16] Luxner, M. H.; Stampfl, J.; Pettermann, H. E. (2007). Numerical simulations of 3D open cell structures - influence of structural irregularities on elasto-plasticity and deformation localization, International Journal of Solids and Structures, Vol. 44, No. 9, 2990-3003, doi:10.1016/j.ijsolstr.2006.08.039

[17] CAESS ProTOp. ProTOp - Topology Optimization Software, from http://caess.eu/, accessed on 03-09-2017

[18] Harl, B.; Predan, J.; Gubeljak, N.; Kegl, M. (2017). On configuration-based optimal design of load-carrying lightweight parts, International Journal of Simulation Modelling, Vol. 16, No. 2, 219-228, doi:10.2507/IJSIMM16(2)3.369

[19] Abaqus/CAE 6.14 User`s guide (2014). Dassault Systèmes Simulia Corp., Providence

[20] Yan, C.; Hao, L.; Hussein, A.; Young, P.; Huang, J.; Zhu, W. (2015). Microstructure and mechanical properties of aluminium alloy cellular lattice structures manufactured by direct metal 
laser sintering, Materials Science and Engineering: A, Vol. 628, 238-246, doi:10.1016/j.msea. $\underline{2015.01 .063}$

[21] Contuzzi, N.; Campanelli, S. L.; Ludovico, A. D. (2011). 3D finite element analysis in the selective laser melting process, International Journal of Simulation Modelling, Vol. 10, No. 3, 113-121, doi:10.2507/IJSIMM10(3)1.169

[22] Ramadani, R.; Pehan, S.; Belšak, A. (2017). Methodology for determining the propagation of vibrations through the gear body, $10^{\text {th }}$ International Conference on Sustainable Energy and Environmental Protection: Modelling and Simulation, 151-161

[23] Allen, R. L.; Mills, D. W. (2004). Signal Analysis: Time, Frequency, Scale, and Structure, IEEE Press, Piscataway, Wiley-Interscience, Hoboken 